\title{
Predictors of Gestational Weight Gain among White and Latina Women and Associations with Birth Weight
}

\author{
Milagros C. Rosal, ${ }^{1}$ Monica L. Wang, ${ }^{2,3}$ Tiffany A. Moore Simas, ${ }^{1}$ Jamie S. Bodenlos, ${ }^{4}$ \\ Sybil L. Crawford, ${ }^{1}$ Katherine Leung, ${ }^{1}$ and Heather Z. Sankey ${ }^{5}$ \\ ${ }^{1}$ Department of Medicine, Division of Preventive and Behavioral Medicine, University of Massachusetts Medical School, \\ 55 Lake Avenue North, Worcester, MA 01655, USA \\ ${ }^{2}$ Boston University School of Public Health, 801 Massachusetts Avenue, Boston, MA 02215, USA \\ ${ }^{3}$ Harvard School of Public Health, 677 Huntington Avenue, Boston, MA 02215, USA \\ ${ }^{4}$ Department of Psychology, Hobart and William Smith Colleges, 217 Gulick Hall, Geneva, NY 14456, USA \\ ${ }^{5}$ Department of Obstetrics and Gynecology, Baystate Medical Center, 759 Chestnut Street, Springfield, MA 01199, USA
}

Correspondence should be addressed to Milagros C. Rosal; milagros.rosal@umassmed.edu

Received 12 May 2016; Accepted 20 June 2016

Academic Editor: Debbie Smith

Copyright ( 2016 Milagros C. Rosal et al. This is an open access article distributed under the Creative Commons Attribution License, which permits unrestricted use, distribution, and reproduction in any medium, provided the original work is properly cited.

\begin{abstract}
This study examined racial/ethnic differences in gestational weight gain (GWG) predictors and association of first-trimester GWG to overall GWG among 271 White women and 300 Latina women. Rates of within-guideline GWG were higher among Latinas than among Whites $(28.7 \%$ versus $24.4 \%, p<0.016)$. Adjusted odds of above-guideline GWG were higher among prepregnancy overweight $(\mathrm{OR}=3.4, \mathrm{CI}=1.8-6.5)$ and obese $(\mathrm{OR}=4.5, \mathrm{CI}=2.3-9.0)$ women than among healthy weight women and among women with above-guideline first-trimester GWG than among those with within-guideline first-trimester $\mathrm{GWG}(\mathrm{OR}=4.9, \mathrm{CI}=$ $2.8-8.8)$. GWG was positively associated with neonate birth size $(p<0.001)$. Interventions targeting prepregnancy overweight or obese women and those with excessive first-trimester GWG are needed.
\end{abstract}

\section{Introduction}

Significant evidence ties gestational weight gain (GWG) to short- and long-term maternal and infant outcomes. To optimize maternal and child health, the Institute of Medicine (IOM) provides guidelines for GWG based on prepregnancy body mass index (BMI) [1]. Greater GWG is recommended for women with prepregnancy BMIs in the underweight (28-40 pounds (lbs), $12.7-18.1 \mathrm{~kg})$ or healthy weight (25$35 \mathrm{lbs}, 11.3-15.9 \mathrm{~kg}$ ) range, with less GWG recommended for prepregnancy overweight (15-25 lbs, $6.8-11.3 \mathrm{~kg}$ ) and obese (11-20 lbs, $5.0-9.1 \mathrm{~kg}$ ) women. However, only 22 to $40 \%$ of women attain GWG within the recommended ranges [2-8], and women of lower socioeconomic status and racial/ethnic minority women have lower adherence to GWG guidelines [5, 9-11]. Among Latina women and depending on national origin, estimates of excessive GWG range from 36 to $51 \%$, whereas estimates of insufficient GWG range from 17 to $30 \%$ $[7,9,10,12,13]$.

Socioeconomic and racial/ethnic disparities in achieving recommended GWG are further compounded by higher pregnancy rates and greater odds of adverse birth-related outcomes among socioeconomically disadvantaged and racial/ethnic minority populations than their more affluent and White counterparts. The pregnancy rate of Latina women in the US is estimated to be two-thirds higher than that of non-Latino Whites [14]. Within the Latina population, nearly half of Caribbean Latina women experience GWG above IOM guidelines [9], and Puerto Rican Latinas are among women with the highest rates of low birth weight neonates [15] and preterm births [16], both predictors of infant mortality [17]. However, little is known about why adherence to guidelines is low among this population. Identifying and understanding factors driving racial/ethnic differences in 
GWG are a priority to target maternal and child health disparities in this growing and at-risk population.

Given the numerous adverse health consequences of excessive and insufficient GWG for the mother and the offspring [1, 4, 18-21], understanding the risk factors for low adherence to IOM-recommended GWG and intervening in at-risk groups are of utmost importance. In targeting interventions, timing of GWG may be important. However, little is known about the influence of early GWG (e.g., first trimester) on overall GWG and other maternal and infant outcomes. A prospective study of a predominantly White female sample indicated that maternal weight change in the first trimester was a stronger predictor of birth weight than weight change in the second or third trimester [22]. However, research on early GWG among Latina women is lacking. The timing and extent of GWG may also be an important determinant of birth weight as well as other maternal and prenatal outcomes; thus, early identification of women who are at risk of excessive or inadequate GWG may be critical to guide the timing and content for intervention delivery to maximize maternal and prenatal health and reduce health disparities.

To address gaps in the literature, this study aimed to examine differences in predictors of gestational weight gain (GWG), assess the association of first-trimester GWG to overall GWG between non-Latina White and Latina women, and examine GWG status with birth outcomes. We hypothesized that women who were overweight or obese before pregnancy would have higher odds of GWG outside of IOM recommendations and that first-trimester GWG status (below, within, or above guideline) would positively correlate with overall GWG.

\section{Methods}

2.1. Participants and Setting. The study's targeted population included non-Latina White and Latina women who received prenatal care from private providers and hospital clinics (i.e., a resident clinic and a midwifery clinic). The study was conducted at Baystate Medical Center, a large tertiary care facility in western Massachusetts with an average of 4,300 deliveries each year, approximately $57 \%$ of them to Latina women (primarily of Puerto Rican origin).

2.2. Procedures. Identification of participants included two screening steps. First, electronic medical record database searches were performed for a retrospective cohort of women who had live deliveries (preterm or full-term) at the medical center from September 1, 2005, to August 31, 2006. Women with multifetal pregnancies, unknown ethnicity, and primary language other than English or Spanish were excluded. A total of 3,966 (of 4,300) patient records met these criteria. Based on estimates of adherence to IOM guidelines in other samples, a sample size of at least 400 women was required for adequate power analysis for the current study. Thus, the second screening step consisted of randomly selecting one quarter $(n=1,016)$ of eligible patient records, stratified by ethnicity (non-Latina White and Latina) and site of prenatal care (hospital clinics and private providers), for additional participant eligibility screening via paper medical chart review. A total of 445 records were excluded. Reasons for exclusion included missing data on prepregnancy weight $(n=226)$ or height $(n=4)$, missing dates of prenatal measurements $(n=138)$, no documentation of prenatal visits in the first trimester of pregnancy $(n=296)$, maternal history of gastric bypass $(n=2)$, or maternal diagnosis of pregestational diabetes $(n=31)$. Of excluded records, $60 \%$ were excluded for one criterion and $40 \%$ were excluded for two or more criteria.

A scannable medical record abstraction form was developed by the research team. The form included fields for recording participant demographics (date of birth, race/ethnicity, primary language, marital status, insurance type, parity, and employment status), psychiatric history (i.e., documented psychiatric diagnosis or use of psychiatric medication), height, and dates and measured weights at each prenatal visit. Three research assistants were trained in the process of data abstraction from paper medical records until $100 \%$ interrater reliability was achieved. Data from completed and cross-checked abstraction forms were scanned and were uploaded into a SAS database.

Data abstraction was performed from 2007 to 2008. During this time frame, revisions of IOM's GWG guidelines were anticipated and were available following data cleaning procedures and at the time of analyses. Thus, the investigative team decided a priori to utilize 2009 guidelines [1] in categorizing GWG measures (described below) with the goal of providing an estimate of likely nonadherence to new recommendations and associated outcomes. Additionally, the 2009 guidelines did not differ greatly from former guidelines yet offered the benefit of a recommended range of gain for obese women in contrast to the previously stated "at least 15 pounds $(6.8 \mathrm{~kg})$ " without an upper bound [17]. All study protocols and procedures were approved by the Baystate Medical Center Institutional Review Board and the University of Massachusetts Medical School Institutional Review Board.

2.3. GWG Measures. Height and prepregnancy weight were obtained from prenatal forms in participants' medical records. Customarily, height is measured by obstetric provider office staff and prepregnancy weight is self-reported by pregnant women at their first prenatal appointment. Prepregnancy BMI was calculated as weight $(\mathrm{kg}) /$ height squared (in meters) and categorized as follows: underweight $(\mathrm{BMI}<$ $\left.18.5 \mathrm{~kg} / \mathrm{m}^{2}\right)$; healthy weight $\left(18.5 \mathrm{~kg} / \mathrm{m}^{2} \leq \mathrm{BMI}<25 \mathrm{~kg} / \mathrm{m}^{2}\right)$; overweight $\left(25 \mathrm{~kg} / \mathrm{m}^{2} \leq \mathrm{BMI}<30 \mathrm{~kg} / \mathrm{m}^{2}\right)$; and obese $\left(30 \mathrm{~kg} / \mathrm{m}^{2} \leq \mathrm{BMI}\right)[17,23]$.

Gestational weight measures were routinely obtained by clinical staff as part of standard obstetric care appointments, as is customary. At each visit, women are weighed and their weight is recorded in prenatal health records, along with gestational age. Each participant's GWG status was determined based on prepregnancy BMI, gestational age, and weight gain at the time of the weight measure. For each prepregnancy weight status category, IOM-recommended trajectories of weight gain were defined (1) in terms of minimum and 
maximum total weight gain at week 13 (end of first trimester) and (2) for subsequent weeks in terms of minimum and maximum weight gain per week. Thus, for each week of gestational age, a minimum and maximum recommended weight gain were calculated.

First-trimester GWG status was determined using the last weight measure recorded during the first trimester. GWG status in the first trimester was assessed by comparing firsttrimester GWG (calculated by subtracting pregravid weight from weight at the last first-trimester prenatal visit) to the IOM-recommended GWG range for gestational age at the last first-trimester prenatal visit. Similarly, GWG status at delivery was determined using weight measured from the last recorded prenatal appointment and was assessed by comparing total GWG (calculated by subtracting pregravid weight from weight at the last prenatal visit prior to delivery) to the IOM-recommended GWG range for gestational age at the last prenatal visit (the average period between the last prenatal visit and delivery is estimated at 6.6 days) [24]. GWG status was categorized as follows: inadequate or "below" if weight gain for gestational age was below the lowest value of the recommended range; appropriate or "within" if weight gain for gestational age was between the recommended range lowest and highest values; and excessive or "above" if weight gain for gestational age was above the highest value of the recommended range.

2.4. Outcome Measures. Gestational age at delivery was calculated based on best dates for estimated date of confinement (EDC). EDC is determined as per clinician evaluation considering concordance of the last menstrual period and firsttrimester ultrasound [25] and documented on the medical record based on clinical care standards. Pregnancies delivered at $<37$ weeks were categorized as preterm and those delivered at $\geq 37$ weeks were full term. Neonate birth weight recorded by nursing staff at the time of delivery was abstracted from the inpatient record. Neonates were categorized as small for gestational age (SGA) and large for gestational age (LGA) if birth weight was $<10$ th and $\geq 90$ th percentile, respectively, of 1999-2000 US national reference data for singleton gestations, accounting for gestational age and gender [26, 27]. Regardless of gestational age, low birth weight (LBW) was defined as $<2,500$ grams [28] and high birth weight (HBW) or macrosomia as $\geq 4,000$ grams [26].

2.5. Statistical Analysis. Descriptive statistics of the study sample stratified by ethnicity were conducted using Chisquare tests or Fisher Exact tests for categorical variables and $t$-tests for continuous variables. Estimated means and standard errors for total GWG were computed for each ethnic group and by prepregnancy weight status category within ethnic group, adjusting for gestational age at the last prenatal visit. Unadjusted associations of GWG status (below, within, or above IOM-recommended range) with participant characteristics were estimated using contingency tables and Chi-square tests. Adjusted associations of GWG status with participant characteristics were estimated using multinomial logistic regression models (within GWG guidelines as the outcome reference category) to allow for the possibility of associations that violated the proportional odds assumption (e.g., a positive association with both above and below GWG guidelines).

Potential effect modification by ethnicity was examined by stratifying contingency tables of GWG status with participant characteristics by ethnicity and by including interaction terms of ethnicity with other predictors in logistic regression models. Model fit was assessed using the Hosmer-Lemeshow goodness-of-fit Chi-square statistic [29]. Infant outcomes were compared by GWG status for the entire group and by ethnicity using contingency tables, Chi-square tests, and logistic regression. Supplemental analyses included conducting backward elimination in the logistic regression analyses to assess whether results were similar after omitting irrelevant or redundant predictors and performing sensitivity analysis comparing results based on the 1990 IOM GWG guidelines versus the 2009 IOM GWG guidelines.

\section{Results}

The final analytic sample included 571 participants $(47 \%$ White and 53\% Latina). The majority of participants were single (64\%) and unemployed (53\%) and had public health insurance $(64 \%)$ (Table 1$)$. Less than half (46\%) of women had prepregnancy BMIs within the healthy weight range, a quarter were obese, and more than half (58\%) exceeded GWG recommendations at the time of delivery. Compared to White women, Latina women were younger and more likely to be single and unemployed, have public insurance, and have higher parity ( $p$ values $<0.05)$. White women had higher prevalence of documented tobacco and alcohol use, were more likely to have a documented psychiatric history, and were more likely to deliver LGA neonates than Latina women $(p$ values $<0.05)$. No other differences by ethnicity were observed. A comparison by prenatal care site revealed that women receiving care in hospital clinics were more likely to be younger, unmarried, unemployed, and nulliparous, have public insurance, have a psychiatric history, and have lower levels of education than those receiving care in private clinics $(p$ values $<0.01)$

Average GWG adjusted for gestational age at delivery was $36.3 \mathrm{lbs}(\mathrm{SE}=0.92)(16.5 \mathrm{~kg}(\mathrm{SE}=0.42))$ for White women and $32.4 \mathrm{lbs}(\mathrm{SE}=0.88)(14.7 \mathrm{~kg}(\mathrm{SE}=0.36))$ for Latina women $(p<0.0001)$. Average GWG by prepregnancy weight status category were as follows: $37.9 \mathrm{lbs}(\mathrm{SE}=2.3)(17.48 \mathrm{~kg}$ $(\mathrm{SE}=1.0))$ for underweight participants; $36.7 \mathrm{lbs}(\mathrm{SE}=0.9)$ $(16.6 \mathrm{~kg}(\mathrm{SE}=0.4))$ for healthy weight participants; $35.3 \mathrm{lbs}$ $(\mathrm{SE}=1.2)(35.3 \mathrm{~kg}(\mathrm{SE}=0.5))$ for overweight participants; and $28.0 \mathrm{lbs}(\mathrm{SE}=1.2)(12.7 \mathrm{~kg}(\mathrm{SE}=0.5))$ for obese participants. Across prepregnancy weight status categories, adherence to IOM GWG recommendations was poor among both ethnic groups, with only $27 \%$ gaining within recommended ranges. Ethnic differences in GWG status at time of delivery for the overall sample were observed, with Latina women less likely to gain in excess than White women $(p=0.016)$ (Figure 1). Latina women were more likely to gain within the IOM-recommended range than White women across all prepregnancy weight status categories, with the exception of the underweight category (among underweight participants, 
TABLE 1: Sample characteristics of overall study sample and by ethnicity $(N=571)$.

\begin{tabular}{|c|c|c|c|c|c|c|c|}
\hline \multirow{3}{*}{ Sample characteristics } & \multicolumn{2}{|c|}{$\begin{array}{l}\text { All women } \\
(n=571)\end{array}$} & \multicolumn{2}{|c|}{$\begin{array}{l}\text { White non-Latina } \\
\qquad(n=271)\end{array}$} & \multicolumn{2}{|c|}{$\begin{array}{l}\text { Latina } \\
(n=300)\end{array}$} & \multirow{3}{*}{$p$ value } \\
\hline & $N$ & $\%$ & $N$ & $\%$ & $N$ & $\%$ & \\
\hline & Mean & $\mathrm{SD}$ & Mean & $\mathrm{SD}$ & Mean & SD & \\
\hline \multicolumn{8}{|l|}{ Demographic factors } \\
\hline \multicolumn{8}{|l|}{ Age category, $N(\%)$} \\
\hline Age 15-19 & 127 & 22.24 & 39 & 14.39 & 88 & 29.33 & $<0.001$ \\
\hline Age $20-24$ & 155 & 27.15 & 64 & 23.62 & 91 & 30.33 & \\
\hline Age 25-29 & 133 & 23.29 & 67 & 24.72 & 66 & 22.00 & \\
\hline Age $30-34$ & 100 & 17.51 & 62 & 22.88 & 38 & 12.67 & \\
\hline Age $\geq 35$ & 56 & 9.81 & 39 & 14.39 & 17 & 5.67 & \\
\hline Mean (SD) & 25.35 & 6.40 & 27.09 & 6.52 & 23.79 & 5.88 & $<0.001$ \\
\hline \multicolumn{8}{|l|}{ Marital status, $N(\%)$} \\
\hline Divorced & 8 & 1.41 & 6 & 2.21 & 2 & 0.67 & $<0.001$ \\
\hline Married & 199 & 35.04 & 129 & 47.60 & 70 & 23.57 & \\
\hline Single & 361 & 63.56 & 136 & 50.18 & 225 & 75.76 & \\
\hline \multicolumn{8}{|l|}{$\begin{array}{l}\text { Employment at onset of pregnancy } \\
N(\%)\end{array}$} \\
\hline Employed & 266 & 46.83 & 153 & 56.67 & 113 & 37.92 & $<0.001$ \\
\hline Not employed & 302 & 53.17 & 117 & 43.33 & 185 & 62.08 & \\
\hline \multicolumn{8}{|l|}{ Parity, $N(\%)$} \\
\hline 0 & 233 & 42.75 & 114 & 44.53 & 119 & 41.18 & 0.003 \\
\hline 1 & 160 & 29.36 & 86 & 33.59 & 74 & 25.61 & \\
\hline 2 & 89 & 16.33 & 39 & 15.23 & 50 & 17.30 & \\
\hline 3 or more & 63 & 11.56 & 17 & 6.64 & 46 & 15.92 & \\
\hline \multicolumn{8}{|l|}{ Behavioral factors } \\
\hline \multicolumn{8}{|l|}{ Alcohol use, $N(\%)$} \\
\hline No & 449 & 78.91 & 193 & 71.22 & 256 & 85.91 & $<0.001$ \\
\hline Yes, past & 112 & 19.68 & 74 & 27.31 & 38 & 12.75 & \\
\hline Yes, this pregnancy & 8 & 1.41 & 4 & 1.48 & 4 & 1.34 & \\
\hline \multicolumn{8}{|l|}{ Tobacco use, $N(\%)$} \\
\hline No & 392 & 68.89 & 172 & 63.47 & 220 & 73.83 & 0.016 \\
\hline Yes, past & 67 & 11.78 & 34 & 12.55 & 33 & 11.07 & \\
\hline Yes, this pregnancy & 110 & 19.33 & 65 & 23.99 & 45 & 15.10 & \\
\hline \multicolumn{8}{|l|}{$\begin{array}{l}\text { Prepregnancy weight categories } \\
\text { (body mass index range), } N(\%)\end{array}$} \\
\hline Underweight $(\mathrm{BMI} \leq 18.4)$ & 33 & 5.78 & 16 & 5.90 & 17 & 5.67 & \\
\hline Normal weight (BMI 18.5-24.9) & 260 & 45.53 & 133 & 49.08 & 127 & 42.33 & 0.315 \\
\hline Overweight (BMI 25.0-29.9) & 138 & 24.17 & 64 & 23.62 & 74 & 24.67 & \\
\hline Obese (BMI $\geq 30.0)$ & 140 & 24.52 & 58 & 21.40 & 82 & 27.33 & \\
\hline \multicolumn{8}{|l|}{ Prenatal care factors } \\
\hline $\begin{array}{l}\text { Week gestation at the 1st prenatal } \\
\text { visit }\end{array}$ & 10.57 & 3.07 & 10.53 & 2.72 & 10.61 & 3.36 & 0.760 \\
\hline Gestational age at the last visit & 35.76 & 5.39 & 35.68 & 5.84 & 35.83 & 4.96 & 0.740 \\
\hline Number of prenatal visits & 9.78 & 3.24 & 9.84 & 3.36 & 9.72 & 3.14 & 0.656 \\
\hline Gestational age at delivery & 38.97 & 5.87 & 38.87 & 6.91 & 39.06 & 4.76 & 0.705 \\
\hline \multicolumn{8}{|l|}{ Prenatal care site, $N(\%)$} \\
\hline Private & 272 & 47.64 & 137 & 50.55 & 135 & 45.00 & 0.185 \\
\hline Hospital clinic & 299 & 52.36 & 134 & 49.45 & 165 & 55.00 & \\
\hline
\end{tabular}


TABle 1: Continued.

\begin{tabular}{|c|c|c|c|c|c|c|c|}
\hline \multirow{3}{*}{ Sample characteristics } & \multicolumn{2}{|c|}{$\begin{array}{c}\text { All women } \\
(n=571)\end{array}$} & \multicolumn{2}{|c|}{$\begin{array}{l}\text { White non-Latina } \\
\qquad(n=271)\end{array}$} & \multicolumn{2}{|c|}{$\begin{array}{l}\text { Latina } \\
(n=300)\end{array}$} & \multirow{3}{*}{$p$ value } \\
\hline & $N$ & $\%$ & $N$ & $\%$ & $N$ & $\%$ & \\
\hline & Mean & $\mathrm{SD}$ & Mean & $\mathrm{SD}$ & Mean & $\mathrm{SD}$ & \\
\hline \multicolumn{8}{|l|}{ Insurance type, $N(\%)$} \\
\hline Commercial/private & 201 & 35.39 & 139 & 51.29 & 62 & 20.88 & $<0.001$ \\
\hline No insurance/unknown & 4 & 0.70 & 4 & 1.48 & 0 & 0.00 & \\
\hline Public & 363 & 63.91 & 128 & 47.23 & 235 & 79.12 & \\
\hline \multicolumn{8}{|c|}{$\begin{array}{l}\text { GWG status with respect to IOM } \\
\text { guidelines }\end{array}$} \\
\hline \multicolumn{8}{|c|}{$\begin{array}{l}\text { GWG status at the last prenatal visit, } \\
N(\%)\end{array}$} \\
\hline Within guidelines & 152 & 26.62 & 66 & 24.35 & 86 & 28.67 & 0.016 \\
\hline Below guidelines & 90 & 15.76 & 33 & 12.18 & 57 & 19.00 & \\
\hline Above guidelines & 329 & 57.62 & 172 & 63.47 & 157 & 52.33 & \\
\hline \multicolumn{8}{|c|}{$\begin{array}{l}\text { GWG status in the 1st trimester, } N \\
(\%)\end{array}$} \\
\hline Within guidelines & 148 & 26.43 & 69 & 26.24 & 79 & 26.60 & 0.537 \\
\hline Above guidelines & 256 & 45.71 & 126 & 47.91 & 130 & 43.77 & \\
\hline Below guidelines & 156 & 27.86 & 68 & 25.86 & 88 & 29.63 & \\
\hline \multicolumn{8}{|l|}{ Psychiatric factors } \\
\hline \multicolumn{8}{|l|}{ Psychiatric history, $N(\%)$} \\
\hline None & 445 & 78.21 & 203 & 75.19 & 242 & 80.94 & 0.003 \\
\hline Anxiety & 15 & 2.64 & 11 & 4.07 & 4 & 1.34 & \\
\hline Depression & 86 & 15.11 & 38 & 14.07 & 48 & 16.05 & \\
\hline Other & 23 & 4.04 & 18 & 6.67 & 5 & 1.67 & \\
\hline \multicolumn{8}{|c|}{ Psychiatric medications, $N(\%)$} \\
\hline No & 539 & 94.40 & 251 & 92.62 & 288 & 96.00 & 0.080 \\
\hline Yes & 32 & 5.60 & 20 & 7.38 & 12 & 4.00 & \\
\hline \multicolumn{8}{|l|}{ Pregnancy outcomes } \\
\hline \multicolumn{8}{|l|}{ Length of pregnancy, $N(\%)$} \\
\hline Term delivery & 504 & 88.73 & 244 & 90.71 & 260 & 86.96 & 0.158 \\
\hline Preterm delivery & 64 & 11.27 & 25 & 9.29 & 39 & 13.04 & \\
\hline \multicolumn{8}{|l|}{ Birth weight parameters } \\
\hline SGA & 56 & 10.04 & 20 & 7.60 & 36 & 12.20 & 0.012 \\
\hline Normal GA & 454 & 81.36 & 212 & 80.61 & 242 & 82.03 & \\
\hline LGA & 48 & 8.60 & 31 & 11.79 & 17 & 5.76 & \\
\hline LBW (<2500 gr) & 53 & 9.45 & 23 & 8.68 & 30 & 10.14 & 0.390 \\
\hline Normal BW & 456 & 81.28 & 213 & 80.38 & 243 & 82.09 & \\
\hline HBW (>4000 gr) & 52 & 9.27 & 29 & 10.94 & 23 & 7.77 & \\
\hline
\end{tabular}

$p$ values are from Chi-square and $t$-tests for ethnic differences.

White women were more likely to have GWG within recommended ranges than Latinas) (Figure 2).

Table 2 presents unadjusted associations between demographics, behavioral factors and psychiatric history, and
GWG status. GWG status was significantly associated with ethnicity, employment status at pregnancy onset, prepregnancy BMI, and first-trimester GWG ( $p$ values $<0.05)$. In logistic regression models, no effect modification by ethnicity 


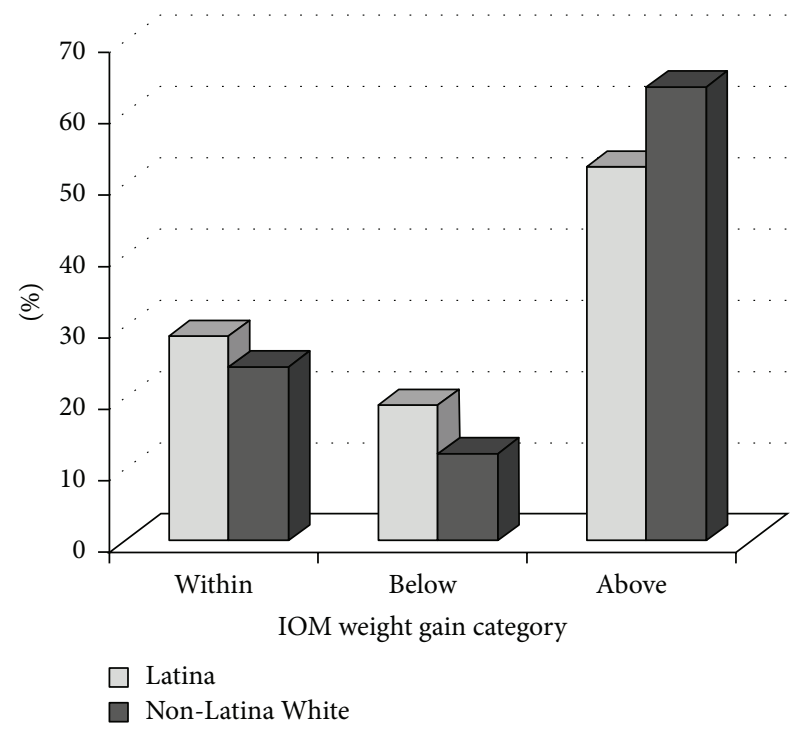

FIGURE 1: Gestational weight gain status among White non-Latina and Latina women.

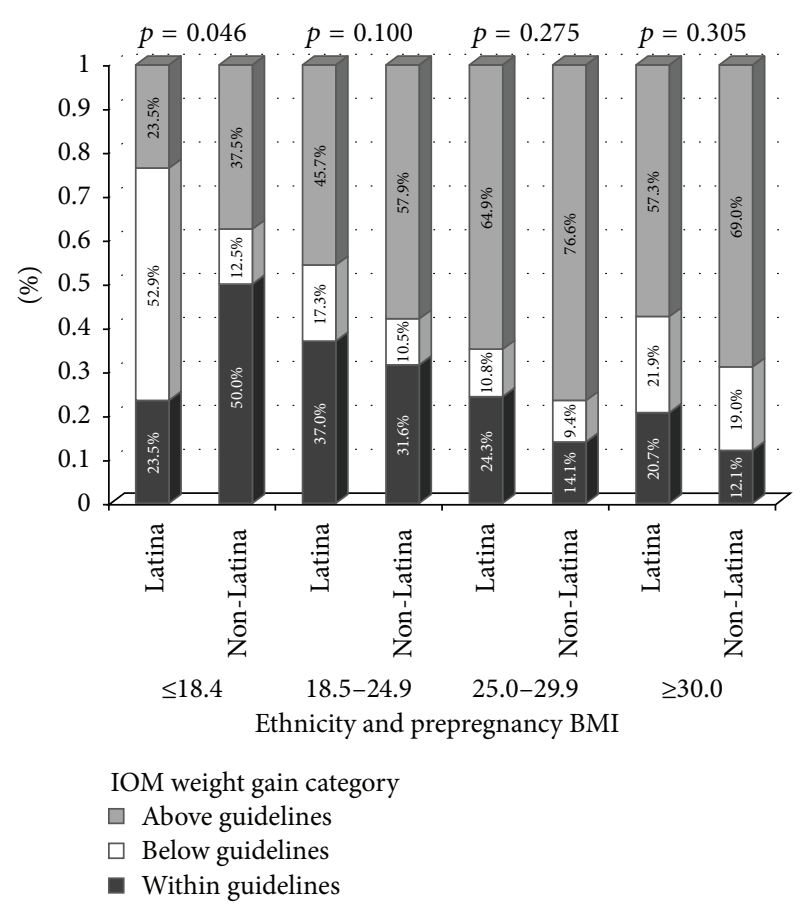

FIGURE 2: Gestational weight gain status by prepregnancy BMI for White non-Latina and Latina women.

was indicated ( $p$ values for interaction terms $>0.05$ ); thus, results are presented for the entire sample. Multivariable logistic regression models estimating participant characteristics associated with GWG status at time of delivery (Table 3) indicated that odds of above-guideline GWG at time of delivery were greater among prepregnancy overweight and obese women compared to healthy weight women $(\mathrm{OR}=$ 3.4, $\mathrm{CI}=1.8-6.5 ; \mathrm{OR}=4.5, \mathrm{CI}=2.3-9.0$, resp.) and among those with first-trimester GWG above guidelines compared to those with GWG within guidelines $(\mathrm{OR}=4.9, \mathrm{CI}=2.8$ 8.8). Odds of below-guideline GWG at time of delivery were greater among prepregnancy underweight and obese women compared to healthy weight $(\mathrm{OR}=5.3, \mathrm{CI}=1.4-20.2$; $\mathrm{OR}=$ $3.5, \mathrm{CI}=1.4-8.7$, resp.) and among women with first-trimester GWG below guidelines compared to within-guideline GWG $(\mathrm{OR}=3.0, \mathrm{CI}=1.3-6.8)$. Odds of below-guideline GWG were lower among women receiving care at hospital clinics compared to those receiving care from a private provider and among past smokers compared to never smokers $(\mathrm{OR}=0.3$, $\mathrm{CI}=0.1-0.9 ; \mathrm{OR}=0.3, \mathrm{CI}=0.1-1.0$, resp.).

A very small number of adverse events were observed within each ethnic group. Thus, Table 4 presents estimates of associations between GWG status and length of pregnancy (preterm versus full-term) and birth weight parameters for the overall sample. GWG status was unrelated to pregnancy length but was associated with birth size (a higher percentage of SGA in pregnancies with below-guideline GWG and a higher percentage of LGA in pregnancies with aboveguideline GWG; $p$ values $<0.05)$. Observed ethnic differences in birth size (Table 1) by which White women were more likely to have LGA neonates and Latina women were more likely to have SGA neonates were not impacted when adjusted for GWG status (data not shown). Supplemental analyses from running more parsimonious models and from sensitivity tests did not yield results that were substantially different from those presented (data not shown).

\section{Discussion}

Findings from this retrospective cohort study provide insights for identifying women at risk for nonadherence to IOM-recommended GWG and for developing targeted interventions. Above-guideline GWG was greater in this cohort (58\%) than in previous studies of multiethnic samples $(35 \%-57 \%$ in prior studies) $[2-4,7]$, suggesting that rates of above-guideline GWG may continue to increase, especially among White women. As noted in other populations [2, 7, 30], prepregnancy weight status predicted GWG in this study. Targeting weight prior to pregnancy is desirable but may be unfeasible for numerous reasons, such as lack of pregnancy intentionality. Targeting weight change during pregnancy may be a more feasible window, as a majority of women seek prenatal care during the first-trimester and are motivated to modify health behaviors [31]. To our knowledge, this is the first study to examine first-trimester GWG status as a predictor of GWG status at time of delivery in a multiethnic sample of women, with first-trimester GWG status predicting overall GWG status among non-Latina White and Latina women. Along with other research [22], study findings indicate that the first trimester of pregnancy may be a critical and feasible window to promote healthy GWG and associated maternal and neonatal outcomes; thus, the identification of women who are at elevated risk for below or above GWG guidelines (e.g., prepregnancy underweight and overweight/obese women) and subsequent delivery of targeted interventions for these subgroups during early prenatal care should be emphasized. 
TABLE 2: Univariate associations of demographic, behavioral, and psychological factors and gestational weight gain status based on 2009 IOM recommendations $(N=571)$.

\begin{tabular}{|c|c|c|c|c|c|c|c|}
\hline \multirow[t]{2}{*}{ Category } & \multicolumn{2}{|c|}{$\begin{array}{l}\text { GWG below } \\
\text { guidelines }\end{array}$} & \multicolumn{2}{|c|}{$\begin{array}{l}\text { GWG within } \\
\text { guidelines }\end{array}$} & \multicolumn{2}{|c|}{$\begin{array}{l}\text { GWG above } \\
\text { guidelines }\end{array}$} & \multirow[t]{2}{*}{$p$ value } \\
\hline & $N$ & $\%$ & $N$ & $\%$ & $N$ & $\%$ & \\
\hline \multicolumn{8}{|l|}{ Age category } \\
\hline Age 15-19 & 26 & 20.47 & 26 & 20.47 & 75 & 59.06 & 0.179 \\
\hline Age $20-24$ & 26 & 16.77 & 43 & 27.74 & 86 & 55.48 & \\
\hline Age 25-29 & 20 & 15.04 & 34 & 25.56 & 79 & 59.40 & \\
\hline Age $30-34$ & 14 & 14.00 & 35 & 35.00 & 51 & 51.00 & \\
\hline Age $\geq 35$ & 4 & 7.14 & 14 & 25.00 & 38 & 67.86 & \\
\hline \multicolumn{8}{|l|}{ Ethnicity } \\
\hline Latina & 57 & 19.00 & 86 & 28.67 & 157 & 52.33 & 0.016 \\
\hline White & 33 & 12.18 & 66 & 24.35 & 172 & 63.47 & \\
\hline \multicolumn{8}{|l|}{ Marital status } \\
\hline Divorced & 1 & 12.50 & 2 & 25.00 & 5 & 62.50 & 0.931 \\
\hline Married & 29 & 14.57 & 57 & 28.64 & 113 & 56.78 & \\
\hline Single & 60 & 16.62 & 93 & 25.76 & 208 & 57.62 & \\
\hline \multicolumn{8}{|c|}{ Employment at onset of pregnancy } \\
\hline Employed & 33 & 12.41 & 82 & 30.83 & 151 & 56.77 & 0.044 \\
\hline Not employed & 55 & 18.21 & 70 & 23.18 & 177 & 58.61 & \\
\hline \multicolumn{8}{|l|}{ Insurance type } \\
\hline Commercial/private & 21 & 10.45 & 64 & 31.84 & 116 & 57.71 & 0.064 \\
\hline No insurance/unknown & 1 & 14.29 & 2 & 28.57 & 4 & 57.14 & \\
\hline Public & 68 & 18.73 & 86 & 23.69 & 209 & 57.58 & \\
\hline \multicolumn{8}{|l|}{ Obstetric provider } \\
\hline Private & 42 & 15.44 & 76 & 27.94 & 154 & 56.62 & 0.793 \\
\hline Hospital clinic & 48 & 16.05 & 76 & 25.42 & 175 & 58.53 & \\
\hline \multicolumn{8}{|l|}{ Parity } \\
\hline 0 & 35 & 15.02 & 57 & 24.46 & 141 & 60.52 & 0.913 \\
\hline 1 & 26 & 16.25 & 42 & 26.25 & 92 & 57.50 & \\
\hline 2 & 13 & 14.61 & 28 & 31.46 & 48 & 53.93 & \\
\hline 3 or more & 11 & 17.46 & 17 & 26.98 & 35 & 55.56 & \\
\hline \multicolumn{8}{|l|}{ Prepregnancy BMI } \\
\hline $\mathrm{BMI} \leq 18.4$ & 11 & 33.33 & 12 & 36.36 & 10 & 30.30 & $<0.001$ \\
\hline BMI 18.5-24.9 & 36 & 13.85 & 89 & 34.23 & 135 & 51.92 & \\
\hline BMI 25.0-29.9 & 14 & 10.14 & 27 & 19.57 & 97 & 70.29 & \\
\hline $\mathrm{BMI} \geq 30.0$ & 29 & 20.71 & 24 & 17.14 & 87 & 62.14 & \\
\hline \multicolumn{8}{|l|}{ Tobacco use } \\
\hline No & 65 & 16.58 & 112 & 28.57 & 215 & 54.85 & 0.236 \\
\hline Yes, past & 8 & 11.94 & 18 & 26.87 & 41 & 61.19 & \\
\hline Yes, this pregnancy & 17 & 15.45 & 21 & 19.09 & 72 & 65.45 & \\
\hline \multicolumn{8}{|l|}{ Alcohol use } \\
\hline No & 68 & 15.14 & 123 & 27.39 & 258 & 57.46 & 0.302 \\
\hline Yes, past & 21 & 18.75 & 23 & 20.54 & 68 & 60.71 & \\
\hline Yes, this pregnancy & 1 & 12.50 & 4 & 50.00 & 3 & 37.50 & \\
\hline \multicolumn{8}{|l|}{ GWG during the 1st trimester } \\
\hline Above & 8 & 3.13 & 43 & 16.80 & 205 & 80.08 & 0.001 \\
\hline Below & 61 & 39.10 & 53 & 33.97 & 42 & 26.92 & \\
\hline Within & 15 & 10.14 & 55 & 37.16 & 78 & 52.70 & \\
\hline
\end{tabular}


TABLE 2: Continued.

\begin{tabular}{|c|c|c|c|c|c|c|c|}
\hline \multirow[t]{2}{*}{ Category } & \multicolumn{2}{|c|}{$\begin{array}{l}\text { GWG below } \\
\text { guidelines }\end{array}$} & \multicolumn{2}{|c|}{$\begin{array}{l}\text { GWG within } \\
\text { guidelines }\end{array}$} & \multicolumn{2}{|c|}{$\begin{array}{l}\text { GWG above } \\
\text { guidelines }\end{array}$} & \multirow[t]{2}{*}{$p$ value } \\
\hline & $N$ & $\%$ & $N$ & $\%$ & $N$ & $\%$ & \\
\hline \multicolumn{8}{|c|}{ Psychiatric diagnosis } \\
\hline None & 71 & 15.96 & 123 & 27.64 & 251 & 56.40 & 0.955 \\
\hline Depression & 13 & 15.12 & 21 & 24.42 & 52 & 60.47 & \\
\hline Anxiety & 3 & 20.00 & 3 & 20.00 & 9 & 60.00 & \\
\hline Other & 3 & 13.04 & 5 & 21.74 & 15 & 65.22 & \\
\hline \multicolumn{8}{|c|}{ Psychiatric medications } \\
\hline No & 86 & 15.96 & 143 & 26.53 & 310 & 57.51 & 0.871 \\
\hline Yes & 4 & 12.50 & 9 & 28.13 & 19 & 59.38 & \\
\hline \multicolumn{8}{|c|}{ Psychiatric history or medications } \\
\hline No & 70 & 16.55 & 117 & 27.66 & 236 & 55.79 & 0.325 \\
\hline Yes & 20 & 13.51 & 35 & 23.65 & 93 & 62.84 & \\
\hline
\end{tabular}

$p$ value is from a Chi-square test.

For both non-Latina White and Latina women in our study sample, maternal smoking status (previous smoker prior to pregnancy) was associated with lower odds of below-recommended GWG which is consistent with previous research indicating that smoking during pregnancy is related to lower GWG and smoking cessation associated with greater GWG $[2,3,32,33]$. Between $29 \%$ and $70 \%$ of women reportedly quit smoking upon becoming pregnant [34]; thus, health care provider attention to smoking history and smoking patterns during pregnancy, with particular focus given to previous or current smokers during early prenatal care, is important to optimize GWG throughout pregnancy.

A larger proportion of SGA infants were born to Latina women than non-Latina White women, with the prevalence of SGA (12.2\%) and preterm delivery (13.0\%) among Latina women in our sample slightly higher than national estimates for Latina women (9\%-10\%) [35]. In contrast, a greater proportion of LGA infants were born to White women. We did not find an association between GWG status at time of delivery and pregnancy length as previously found [36]. In addition, we did not find ethnic differences in low or high birth weight, which is in contrast to prior data indicating that Puerto Rican Latinas have some of the highest rates of low birth weight neonates [15] and preterm births [16] in the US. Multiple factors not assessed in this retrospective cohort study (e.g., prior preterm births, gestational diabetes mellitus) may contribute to and account for differences in birth outcomes observed in this study compared to previous studies. In addition, conventional measures of GWG may introduce bias when studying GWG-preterm birth associations [37]. Additional studies with larger, ethnically diverse samples are needed to elucidate predictors driving racial/ethnic disparities in birth weight outcomes.

Study strengths include the sample's ethnic and socioeconomic diversity (i.e., White/Latina women, public/commercial insurance, and hospital clinics/private provider) and inclusion of women who delivered pre- and full-term (previous studies have been limited to women who delivered full-term) [2, 7]. Although no data were available on place of birth, most Latinos in the region where the study was conducted are of Puerto Rican descent, a largely understudied population with considerable health disparities, including infant mortality [38].

Study limitations include the retrospective study design and the use of existing medical record data (with data gathered within the context of clinical activities rather than by trained research staff). However, all providers completed similar maternal and prenatal medical forms, which were routinely filed in the hospital medical record database prior to delivery. Participants' self-reported prepregnancy weight (as opposed to prepregnancy weight measured in a clinical or research setting) was used to determine GWG status. However, the IOM guidelines are based on studies that similarly use self-reported prepregnancy weight [39], and self-reported prepregnancy weight has been found to be highly correlated with clinically measured weight [40-42]. Information available on smoking patterns during pregnancy (i.e., number of cigarettes, quit date) was restricted. Furthermore, smoking status data was collected in the context of the first prenatal appointment and may be subject to social desirability bias and may only reflect smoking status at the first prenatal visit. However, the prevalence of smoking in our sample (19\%) is consistent with smoking rates among White [2, 3, 32, 33] and Latina pregnant women [43] in previous studies. Presence of gestational diabetes, shown to be associated with birth weight $[44,45]$, was not controlled for. Women without a first-trimester prenatal visit and with missing prepregnancy BMI data were excluded from analysis; as systematic biases might exist between women who were or were not missing these data, findings may not be representative of the larger population from which the study sample was drawn. Study findings may not be generalizable to other (non-Puerto Rican) Latino subgroups. Lastly, the study 
TABLE 3: Multivariate analysis of predictors of gestational weight gain status in overall study sample $(N=571)$.

\begin{tabular}{|c|c|c|c|c|}
\hline \multirow[t]{2}{*}{ Variable } & \multicolumn{2}{|c|}{$\begin{array}{l}\text { Adjusted OR for GWG } \\
\text { below guidelines }\end{array}$} & \multicolumn{2}{|c|}{$\begin{array}{l}\text { Adjusted OR for GWG } \\
\text { above guidelines }\end{array}$} \\
\hline & Odds ratio $(95 \% \mathrm{CI})$ & $p$ value & Odds ratio $(95 \% \mathrm{CI})$ & $p$ value \\
\hline \multicolumn{5}{|c|}{ GWG during the 1st trimester } \\
\hline Within guidelines & Reference & $<0.0001$ & & $<0.0001$ \\
\hline Above guidelines & $0.29(0.08-1.01)$ & & $4.92(2.75-8.81)$ & \\
\hline Below guidelines & $3.01(1.33-6.81)$ & & $0.39(0.21-0.74)$ & \\
\hline \multicolumn{5}{|l|}{ Prepregnancy BMI } \\
\hline $18.5-24.9$ & Reference & 0.0072 & Reference & $<0.0001$ \\
\hline$\leq 18.4$ & $5.26(1.37-20.17)$ & & $0.19(0.06-0.58)$ & \\
\hline $25.0-29.9$ & $0.83(0.27-2.58)$ & & $3.44(1.82-6.50)$ & \\
\hline$\geq 30.0$ & $3.47(1.38-8.70)$ & & $4.55(2.29-9.04)$ & \\
\hline \multicolumn{5}{|l|}{ Ethnicity } \\
\hline White & Reference & 0.9446 & Reference & 0.0069 \\
\hline Latina & $0.97(0.43-2.17)$ & & $0.46(0.26-0.81)$ & \\
\hline \multicolumn{5}{|l|}{ Tobacco use } \\
\hline No & Reference & 0.0402 & Reference & 0.2008 \\
\hline Yes, past & $0.26(0.07-0.99)$ & & $1.09(0.49-2.42)$ & \\
\hline Yes, this pregnancy & $1.77(0.64-4.84)$ & & $2.01(0.93-4.31)$ & \\
\hline \multicolumn{5}{|l|}{ Alcohol use } \\
\hline No & Reference & 0.5637 & Reference & 0.1978 \\
\hline Yes, past & $1.69(0.64-4.49)$ & & $1.15(0.57-2.30)$ & \\
\hline Yes, this pregnancy & $0.85(0.05-13.18)$ & & $0.15(0.02-1.29)$ & \\
\hline \multicolumn{5}{|l|}{ Age group } \\
\hline $25-29$ & Reference & 0.2141 & Reference & 0.1470 \\
\hline $15-19$ & 3.07 (0.87-10.91) & & $1.50(0.61-3.67)$ & \\
\hline $20-24$ & $1.35(0.45-4.04)$ & & $0.83(0.40-1.74)$ & \\
\hline $30-34$ & $0.60(0.20-1.76)$ & & $0.52(0.25-1.08)$ & \\
\hline$\geq 35$ & $0.50(0.10-2.44)$ & & $1.18(0.49-2.86)$ & \\
\hline \multicolumn{5}{|l|}{ Insurance } \\
\hline Commercial/private & Reference & 0.3929 & Reference & 0.1126 \\
\hline Public & $1.58(0.55-4.57)$ & & $1.75(0.88-3.50)$ & \\
\hline \multicolumn{5}{|l|}{ Employment at onset } \\
\hline Employed & Reference & 0.2533 & Reference & 0.2683 \\
\hline Not employed & $1.63(0.71-3.76)$ & & $1.39(0.77-2.50)$ & \\
\hline \multicolumn{5}{|l|}{ Parity } \\
\hline 0 & Reference & 0.9476 & Reference & 0.5305 \\
\hline 1 & $0.93(0.38-2.29)$ & & $0.94(0.51-1.74)$ & \\
\hline 2 & $1.24(0.42-3.61)$ & & $0.73(0.34-1.61)$ & \\
\hline 3 or more & $1.28(0.35-4.59)$ & & $0.52(0.20-1.30)$ & \\
\hline \multicolumn{5}{|l|}{ Obstetric provider } \\
\hline Private & Reference & 0.0255 & Reference & 0.3441 \\
\hline Hospital clinic & $0.35(0.14-0.89)$ & & $0.75(0.41-1.37)$ & \\
\hline \multicolumn{5}{|l|}{ Psychiatric medications } \\
\hline No & Reference & 0.6482 & Reference & 0.2837 \\
\hline Yes & $0.67(0.12-3.72)$ & & $0.51(0.15-1.73)$ & \\
\hline \multicolumn{5}{|l|}{ Psychiatric diagnosis } \\
\hline None & Reference & 0.6362 & Reference & 0.5163 \\
\hline Anxiety & $2.08(0.25-17.06)$ & & $1.26(0.25-6.50)$ & \\
\hline Depression & $1.14(0.37-3.52)$ & & $1.27(0.60-2.67)$ & \\
\hline Other & $3.16(0.42-23.92)$ & & $3.57(0.66-19.31)$ & \\
\hline
\end{tabular}

was not adequately powered to examine ethnic differences in pregnancy outcomes by GWG status; thus, results of GWG associated with outcomes of interest by ethnicity are exploratory.
Understanding factors that contribute to inadequate and excessive GWG is critical to the development of interventions that seek to optimize recommended GWG. Additional researches on racial/ethnic differences in the influence of 
TABLE 4: Prevalence of selected pregnancy outcomes by gestational weight status in overall study sample $(N=571)$.

\begin{tabular}{lccc}
\hline \multirow{2}{*}{ Outcomes } & \multicolumn{3}{c}{ Gestational weight gain status } \\
& Below & Within & Above \\
\hline Length of pregnancy $(p=0.966)^{1}$ & & & \\
$\quad$ Term delivery & $80(88.9)$ & $134(88.2)$ & $290(89.0)$ \\
Preterm delivery & $10(11.1)$ & $18(11.8)$ & $36(11.0)$ \\
\hline Birth weight $(p=0.001)^{1}$ & & & \\
SGA & $18(20.7)$ & $15(10.1)$ & $23(7.1)$ \\
Normal GA & $66(75.9)$ & $124(83.2)$ & $264(82.0)$ \\
LGA & $3(3.4)$ & $10(6.7)$ & $35(10.9)$ \\
\hline Birth weight $(p=0.061)^{1}$ & & & \\
LBW $(<2500)$ & $10(11.5)$ & $18(12.1)$ & $25(7.7)$ \\
Normal BW & $73(83.9)$ & $122(81.9)$ & $261(80.3)$ \\
HBW $(>4000)$ & $4(4.6)$ & $9(6.0)$ & $39(12.0)$ \\
\hline
\end{tabular}

${ }^{1} p$ value for association between GWG status and selected pregnancy outcomes in the entire sample.

GA: gestational age; SGA: small for gestational age; LGA: large for gestational age; BW: birth weight; LBW: low birth weight; HBW: high birth weight.

early GWG on GWG and other maternal and neonatal outcomes are needed to guide the development of interventions tailored for socioeconomically and ethnically diverse populations.

\section{Additional Points}

Implications for Practice and/or Policy. Study findings highlight the importance of identifying and targeting populations at high risk for excessive GWG, particularly in early pregnancy. Emphasizing early prenatal care and facilitating adherence to GWG recommendations in the first trimester are particularly relevant among prepregnancy underweight and overweight/obese women. Within the clinical setting, identifying populations at risk for both above- and belowguideline GWG during early prenatal care is critical for optimizing GWG. Timely targeted interventions are needed for health care providers and practitioners to deliver throughout pregnancy with the ultimate goal of improving maternal and neonatal short- and long-term outcomes.

\section{Competing Interests}

The authors have no competing financial interests to declare.

\section{Acknowledgments}

This study was funded by the Division of General Obstetrics \& Gynecology Research Fund, Baystate Medical Center, Springfield, MA, USA.

\section{References}

[1] Institute of Medicine, Weight Gain During Pregnancy: Reexamining the Guidelines, National Academic Press, Washington, DC, USA, 2009.
[2] C. S. Wells, R. Schwalberg, G. Noonan, and V. Gabor, "Factors influencing inadequate and excessive weight gain in pregnancy: Colorado, 2000-2002," Maternal and Child Health Journal, vol. 10, no. 1, pp. 55-62, 2006.

[3] C. M. Olson and M. S. Strawderman, "Modifiable behavioral factors in a biopsychosocial model predict inadequate and excessive gestational weight gain," Journal of the American Dietetic Association, vol. 103, no. 1, pp. 48-54, 2003.

[4] N. E. Stotland, Y. W. Cheng, L. M. Hopkins, and A. B. Caughey, "Gestational weight gain and adverse neonatal outcome among term infants," Obstetrics and Gynecology, vol. 108, no. 3, pp. 635643, 2006.

[5] B. E. Gould Rothberg, U. Magriples, T. S. Kershaw, S. S. Rising, and J. R. Ickovics, "Gestational weight gain and subsequent postpartum weight loss among young, low-income, ethnic minority women," American Journal of Obstetrics and Gynecology, vol. 204, no. 1, pp. 52.e1-52.e11, 2011.

[6] C. M. Olson, M. S. Strawderman, P. S. Hinton, and T. A. Pearson, "Gestational weight gain and postpartum behaviors associated with weight change from early pregnancy to 1 y postpartum," International Journal of Obesity and Related Metabolic Disorder, vol. 27, no. 1, pp. 117-127, 2003.

[7] P. Brawarsky, N. E. Stotland, R. A. Jackson et al., "Pre-pregnancy and pregnancy-related factors and the risk of excessive or inadequate gestational weight gain," International Journal of Gynecology and Obstetrics, vol. 91, no. 2, pp. 125-131, 2005.

[8] S. Y. Chu, S. Y. Kim, and C. L. Bish, "Prepregnancy obesity prevalence in the United States, 2004-2005," Maternal and Child Health Journal, vol. 13, no. 5, pp. 614-620, 2009.

[9] L. Chasan-Taber, M. D. Schmidt, P. Pekow, B. Sternfeld, C. G. Solomon, and G. Markenson, "Predictors of excessive and inadequate gestational weight gain in Hispanic women," Obesity, vol. 16, no. 7, pp. 1657-1666, 2008.

[10] H. Sangi-Haghpeykar, K. Lam, and S. P. Raine, "Gestational weight gain among hispanic women," Maternal and Child Health Journal, vol. 18, no. 1, pp. 153-160, 2014.

[11] I. E. Headen, E. M. Davis, M. S. Mujahid, and B. Abrams, "Racial-ethnic differences in pregnancy-related weight," Advances in Nutrition, vol. 3, no. 1, pp. 83-94, 2012.

[12] L. O. Walker, M. M. Hoke, and A. Brown, "Risk factors for excessive or inadequate gestational weight gain among Hispanic women in a U.S.-Mexico border state," Journal of Obstetric, Gynecologic, and Neonatal Nursing, vol. 38, no. 4, pp. 418-429, 2009.

[13] A. M. Siega-Riz and C. J. Hobel, "Predictors of poor maternal weight gain from baseline anthropometric, psychosocial, and demographic information in a hispanic population," Journal of the American Dietetic Association, vol. 97, no. 11, pp. 1264-1268, 1997.

[14] S. J. Ventura, J. C. Abma, W. D. Mosher, and S. K. Henshaw, "Estimated pregnancy rates by outcome for the United States, 1990-2004," National Vital Statistics Report, vol. 56, no. 15, pp. $1-28,2008$.

[15] T. J. Rosenberg, T. P. Raggio, and M. A. Chiasson, "A further examination of the 'epidemiologic paradox': birth outcomes among Latinas," Journal of the National Medical Association, vol. 97, no. 4, pp. 550-556, 2005.

[16] C. R. Stein, D. A. Savitz, T. Janevic et al., "Maternal ethnic ancestry and adverse perinatal outcomes in New York City," American Journal of Obstetrics and Gynecology, vol. 201, no. 6, pp. 584.el-584.e9, 2009. 
[17] Institute of Medicine, Subcommittee on Nutritional Status, and Weight Gain during Pregnancy, Nutrition During Pregnancy, No. 27, National Academy Press, Washington, DC, USA, 1990.

[18] E. Oken, E. M. Taveras, K. P. Kleinman, J. W. Rich-Edwards, and M. W. Gillman, "Gestational weight gain and child adiposity at age 3 years," American Journal of Obstetrics and Gynecology, vol. 196, no. 4, pp. 322.el-322.e8, 2007.

[19] S. Y. Chu, W. M. Callaghan, C. L. Bish, and D. D’Angelo, "Gestational weight gain by body mass index among US women delivering live births, 2004-2005: fueling future obesity," American Journal of Obstetrics and Gynecology, vol. 200, no. 3, pp. 271.e1-271.e7, 2009.

[20] Y. Linné, L. Dye, B. Barkeling, and S. Rössner, "Long-term weight development in women: a 15-year follow-up of the effects of pregnancy," Obesity Research, vol. 12, no. 7, pp. 1166-1178, 2004.

[21] A. R. Amorim, S. Rössner, M. Neovius, P. M. Lourenço, and Y. Linné, "Does excess pregnancy weight gain constitute a major risk for increasing long-term BMI?" Obesity, vol. 15, no. 5, pp. 1278-1286, 2007.

[22] J. E. Brown, M. A. Murtaugh, D. R. Jacobs Jr., and H. C. Margellos, "Variation in newborn size according to pregnancy weight change by trimester," American Journal of Clinical Nutrition, vol. 76, no. 1, pp. 205-209, 2002.

[23] K. M. Rasmussen and A. L. Yaktine, Eds., Weight Gain during Pregnancy: Re-Examining the Guidelines, National Academy Press, Washington, DC, USA, 2009.

[24] T. A. Moore Simas, D. K. Doyle Curiale, J. Hardy, S. Jackson, Y. Zhang, and X. Liao, "Efforts needed to provide institute of medicine-recommended guidelines for gestational weight gain," Obstetrics \& Gynecology, vol. 115, no. 4, pp. 777-783, 2010.

[25] American College of Obstetricians and Gynecologists, "ACOG practice Bulletin No. 101: ultrasonography in pregnancy," Obstetrics and Gynecology, vol. 113, no. 2, part 1, pp. 451-461, 2009.

[26] American College of Obstetricians and Gynecologists (ACOG), "Intrauterine growth restriction," ACOG Practice Bulletin 12, American College of Obstetricians and Gynecologists (ACOG), Washington, DC, USA, 2000.

[27] C. E. Margerison Zilko, D. Rehkopf, and B. Abrams, "Association of maternal gestational weight gain with short- and longterm maternal and child health outcomes," American Journal of Obstetrics and Gynecology, vol. 202, no. 6, pp. 574.el-574.e8, 2010.

[28] World Health Organization (WHO), International Statistical Classification of Diseases and Related Health Problems, Tenth Revision, World Health Organization, Geneva, Switzerland, 1992.

[29] D. W. Hosmer and S. Lemeshow, Applied Logistic Regression, John Wiley \& Sons, New York, NY, USA, 2nd edition, 2000.

[30] N. E. Stotland, J. S. Haas, P. Brawarsky, R. A. Jackson, E. FuentesAfflick, and G. J. Escobar, "Body mass index, provider advice, and target gestational weight gain," Obstetrics \& Gynecology, vol. 105, no. 3, pp. 633-638, 2005.

[31] J. A. Martin, B. E. Hamilton, P. D. Sutton et al., "Births: final data for 2007," National Vital Statistics Reports, vol. 58, no. 24, pp. 1-85, 2010.

[32] J. P. Furuno, L. Gallicchio, and M. Sexton, "Cigarette smoking and low maternal weight gain in medicaid-eligible pregnant women," Journal of Women's Health, vol. 13, no. 7, pp. 770-777, 2004.
[33] A. S. Olafsdottir, G. V. Skuladottir, I. Thorsdottir, A. Hauksson, and L. Steingrimsdottir, "Combined effects of maternal smoking status and dietary intake related to weight gain and birth size parameters," British Journal of Obstetrics and Gynaecology, vol. 113, no. 11, pp. 1296-1302, 2006.

[34] A report of the Surgeon General, "Tobacco smoke causes disease: The biology and behavioral basis for smoking attributable disease," http://www.mchb.hrsa.gov/whusall/hstat/hsrmh/ pages/228sdp.html.

[35] Centers for Disease Control and Prevention and National Vital Statistics System, "Annual natality files," http://www.cdc.gov/ nchs/births.htm.

[36] M. Viswanathan, A. M. Siega-Riz, M. K. Moos et al., "Outcomes of maternal weight gain," Evidence Report/Technology Assessment (Full Report) 168, 2008.

[37] J. A. Hutcheon, L. M. Bodnar, K. S. Joseph, B. Abrams, H. N. Simhan, and R. W. Platt, "The bias in current measures of gestational weight gain," Paediatric and Perinatal Epidemiology, vol. 26, no. 2, pp. 109-116, 2012.

[38] MassCHIP, Race/Hispanic Ethnicity Report: Birth and Perinatal Indicators, Massachusetts Department of Public Health, Boston, Mass, USA, 2010.

[39] A. M. Siega-Riz, L. S. Adair, and C. J. Hob, "Institute of medicine maternal weight gain recommendations and pregnancy outcome in a predominantly hispanic population," Obstetrics and Gynecology, vol. 84, no. 4, pp. 565-573, 1994.

[40] S. Avishai-Eliner, K. L. Brunson, C. A. Sandman, and T. Z. Baram, "Stressed-out, or in (utero)?" Trends in Neurosciences, vol. 25, no. 10, pp. 518-524, 2002.

[41] C. Stevens-Simon, K. J. Roghmann, and E. R. McAnarney, "Relationship of self-reported prepregnant weight and weight gain during pregnancy to maternal body habitus and age," Journal of the American Dietetic Association, vol. 92, no. 1, pp. 85-87, 1992.

[42] C. Stevens-Simon, E. R. McAnarney, and M. P. Coulter, "How accurately do pregnant adolescents estimate their weight prior to pregnancy?" Journal of Adolescent Health Care, vol. 7, no. 4, pp. 250-254, 1986.

[43] A. E. Haskins, E. R. Bertone-Johnson, P. Pekow, E. Carbone, R. T. Fortner, and L. Chasan-Taber, "Smoking during pregnancy and risk of abnormal glucose tolerance: a prospective cohort study," BMC Pregnancy and Childbirth, vol. 10, p. 55, 2010.

[44] G. Seghieri, R. Anichini, A. De Bellis, L. Alviggi, F. Franconi, and M. C. Breschi, "Relationship between gestational diabetes mellitus and low maternal birth weight," Diabetes Care, vol. 25, no. 10, pp. 1761-1765, 2002.

[45] B. Krstevska, S. Mishevska, E. Janevska et al., "Gestational Diabetes Mellitus - the impact of maternal body mass index and glycaemic control on baby's birth weight," Prilozi, vol. 30, no. 2, pp. 115-124, 2009. 


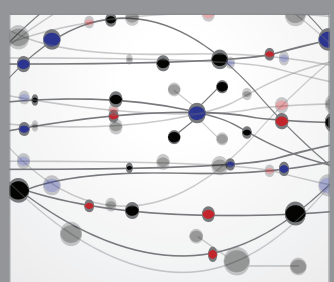

The Scientific World Journal
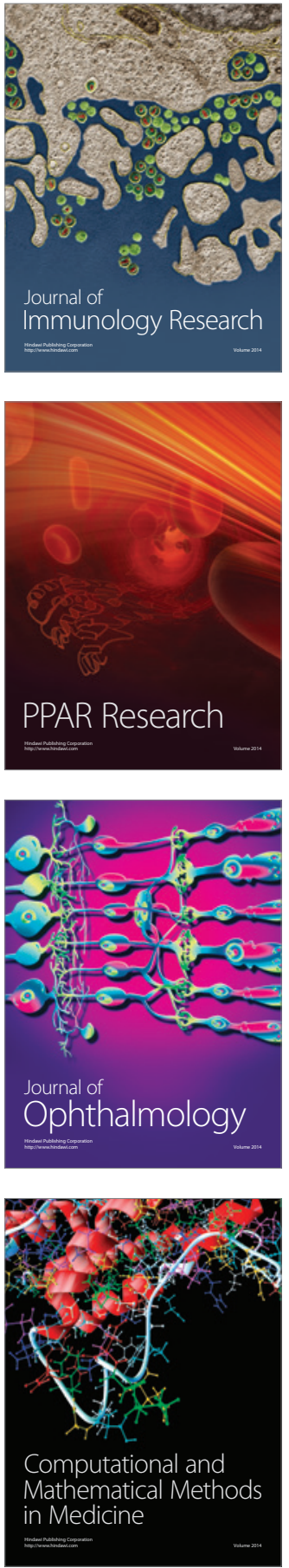

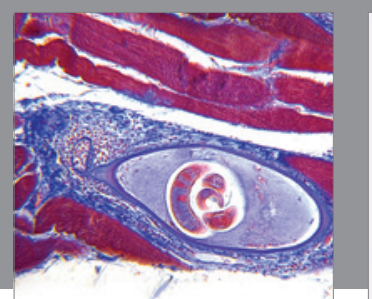

Gastroenterology Research and Practice

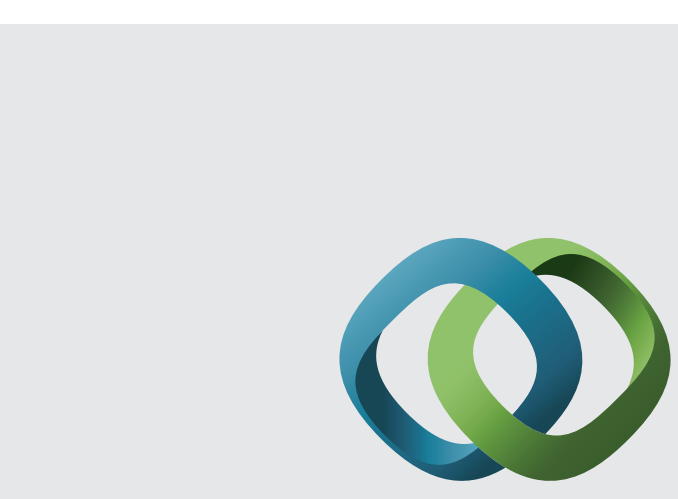

\section{Hindawi}

Submit your manuscripts at

http://www.hindawi.com
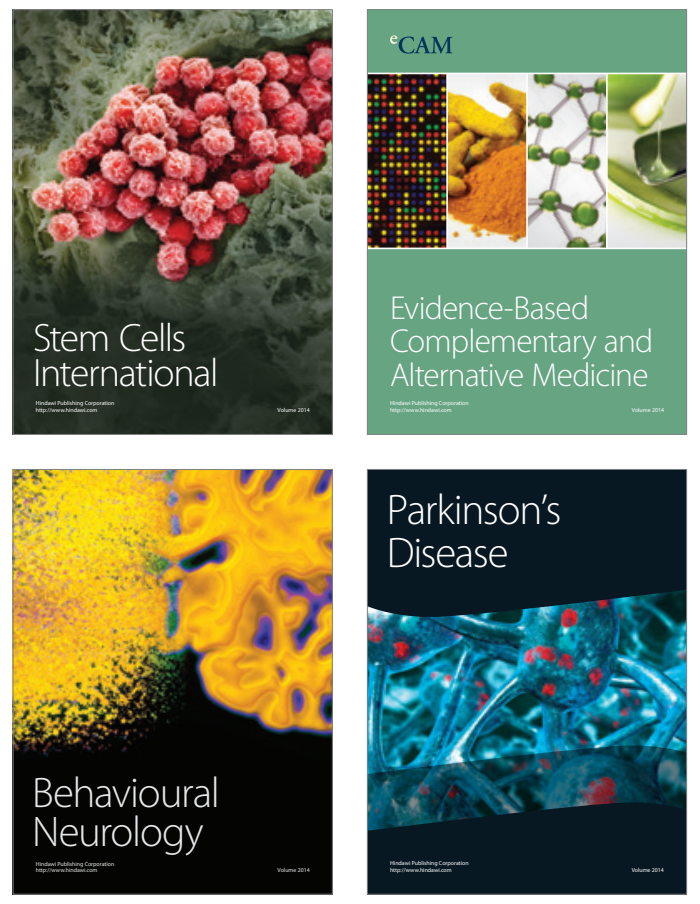
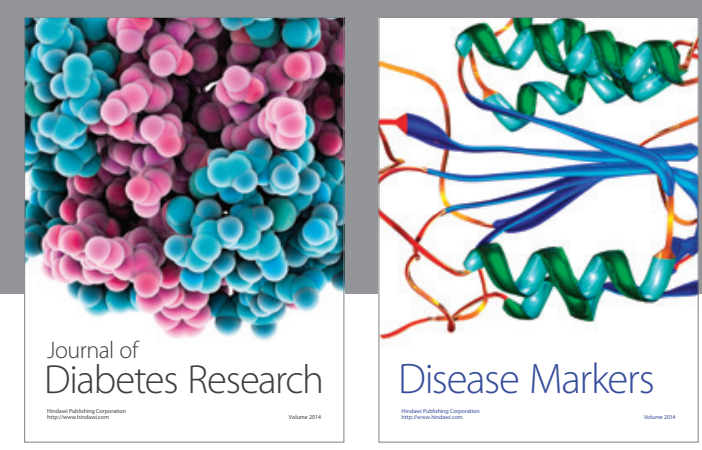

Disease Markers
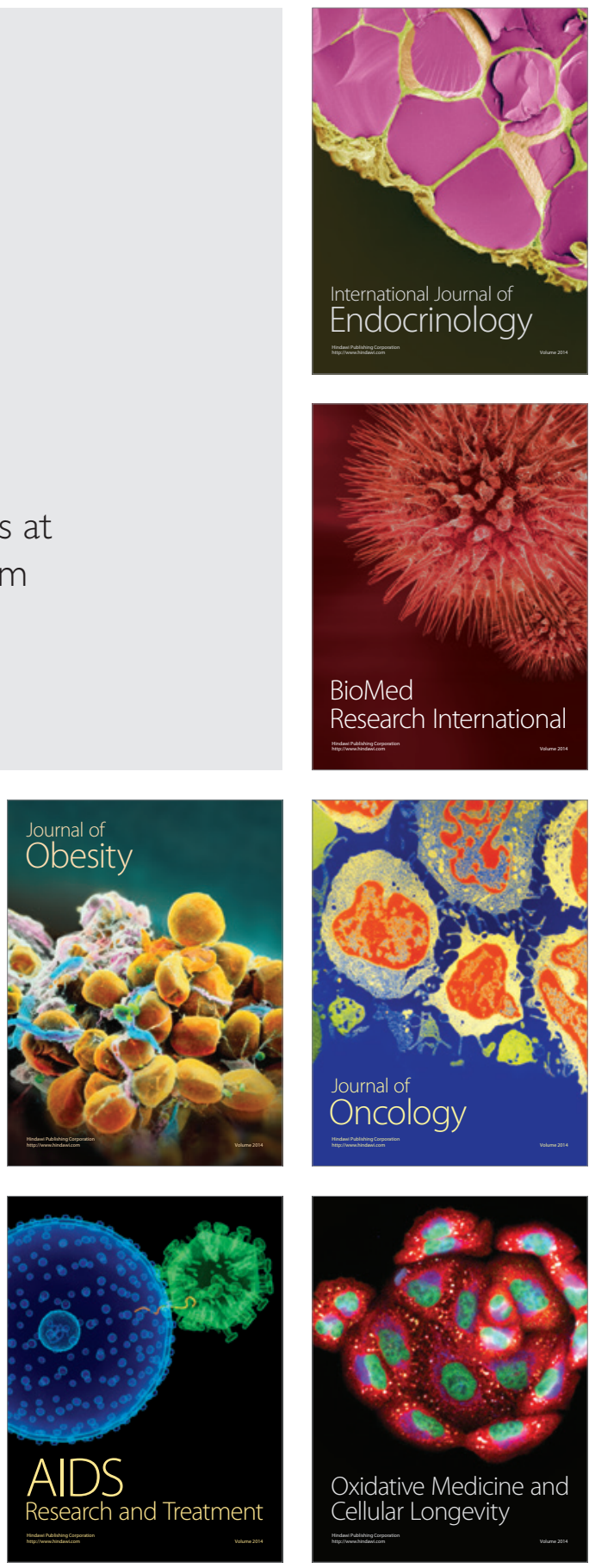